in his law of the rectilinear diameter. Combining this with Guldberg's finding ${ }^{1}$ that the critical density is four times the density of a substance at absolute zero, Davies ${ }^{2}$ has shown that

$$
\alpha=\frac{\mathrm{I}}{2 \mathrm{~T}_{c}-\mathrm{T}}
$$

where $\alpha$ is the coefficient of cubic expansion of the liquid. This would lead to a critical temperature for mercury above $2600^{\circ}$.

One may also employ the equivalent but older relationship of Thorpe and Rücker ${ }^{3}$

$$
\mathrm{T}_{c}=\frac{\mathrm{T}_{2} s_{1}-\mathrm{T}_{1} s_{2}}{\mathrm{~A}\left(s_{1}-s_{2}\right)}
$$

where $s_{1}$ and $s_{2}$ are densities of the liquid at the temperatures $T_{1}$ and $T_{2}$. Young gives a value I.974 for the constant A, and states that for twentyfour substances for which the required data are accurately known, the discordances only in a few cases exceed $2 \%$. This formula leads also to a critical temperature for mercury above $2600^{\circ}$.

The rule of Guldberg ${ }^{4}$ connecting critical temperature and boiling point is clearly inapplicable here, as it rests ultimately on the assumption that the critical pressures of all substances are nearly identical.

obhilin College, Oberlin, Ohio.

\title{
THE MECHANICAL STIMULUS TO CRYSTALLIZATION. ${ }^{5}$
}

By S. W. Young and W. J. Van SickLEN.

Received July 1, 1913.

In two previous papers on this subject ${ }^{6}$ it has been shown that crystallization in supercooled liquids may be forced at any temperature below their melting points. In other words, there is no so-called metastable field, in which crystallization can only be stimulated by the addition of a crystal of the new phase which is to appear. Since the publication of these papers there has appeared a most interesting paper by the Earl of Berkeley, ${ }^{7}$ in which the matter of the metastable limit is taken up from the theoretical point of view in a very clear manner. Basing the theoretical necessity for the existence of a metastable limit on the van der Waals type of doubly flexed curve, which he shows is likewise applicable to solutions, he seeks for some explanation which will harmonize the theory

1 Chem. Zentr., 1898, p. 1042.

2 Phil. Mag., [6] 23, 657 (I912).

3 $J$. Chem. Soc., 45, 135 (1884).

I. physik. Chem., 5, 374 (1890).

${ }^{5}$ Abstracted from a Thesis for the Degree of Master of Arts, presented by Mr. W. J. Van Sicklen to the Faculty of Stanford University, December, I9I2.

6 This JOURNAL, 33, I48, I375.

7 Phil. Mag., Aug., 1912, 254. 
with the facts. This he finds in the assumption that as a result of the shock produced in the unstable liquid, alternate compression and rarefaction waves are set up, and that these are competent to carry the system past the metastable limit (theoretical), provided they are sufficiently powerful. He furthermore cites results on cane sugar solutions which were forced to crystallize within three degrees of their saturation temperature, whereas by shaking with hard bodies they required a supercooling of six and one-half degrees. His experimental results therefore confirm the point of view taken in the previous papers mentioned, to the effect that there is no such thing as a metastable limit in the sense in which that term has been commonly used, that is, as a field in which the only way to start crystallization is by the introduction of a fragment of the new phase to be formed. The fact that the theoretical van der Waals type of curve is not necessarily invalidatep by this fact, is most interesting, and the Earl

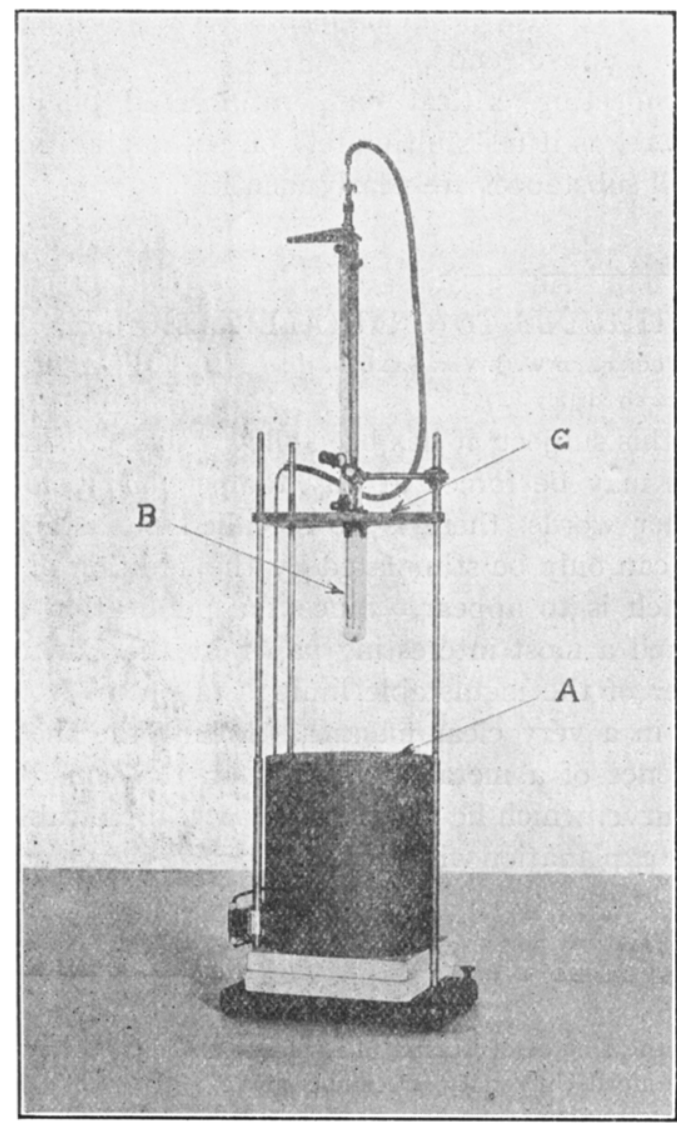

Fig. I. of Berkeley's theoretical treatment of the case seems likely to prove of considerable value in the further investigation of the problem.

In the meantime, we have busied ourselves with the further perfection of the impact apparatus shown in the first of the above papers, and with attempts to establish a more accurate quantitative relationship between the value of the impact and the supercooling. In the course of this work, some interesting facts have come to light which it is the purpose of this paper to describe.

The Apparatus. - The apparatus used in this work was an elaboration and refinement of the one previously employed. In designing it, the object was to make it as near to a precision instrument as possible with the knowl- 
edge at hand. The essential part of the apparatus is shown in Fig. I. $A$ is the constant temperature bath, consisting of a carefully insulated and covered battery jar. For cooling to below zero a mixture of ice, water and alcohol was found very satisfactory. The temperature could be lowered by the addition of alcohol and raised by the addition of water. With a little attention occasionally, the temperature could be kept constant for several hours, to within less than one-hundredth of a degree.

A carriage, $C$, of heavy cast brass held the impact parts, together with the tube containing the water to be studied, and also supported the scale and vernier for measuring the drop distance of the hammer. A reading microscope was attached to assist in these readings. This carriage was arranged to slide up and down on the three upright rods, so that at any time the vessel carrying the water could be removed and replaced, or ice which had formed in it could be melted. The large test tube for containing the water is shown at $B$, and within it may be seen the impact parts and the thermometer.

Fig. 2 is an enlarged drawing of the impact parts. Through the sleeve $S$ works the hammer $H$, in bearings as near frictionless as possible. The im- $H$ pact is made by the fall of $H$ upon the anvil $A$, the energy of the impact being determined by the weight of the hammer and the distance of the drop. The weight of the hammer may be varied by means of steel caps of different sizes, which may be screwed onto the top of the hammer rod. The hammer was held in the raised position by means of a vacuum cap, and was released at the desired time by admitting air to the cap. This form of release seems to operate quite satisfactorily.

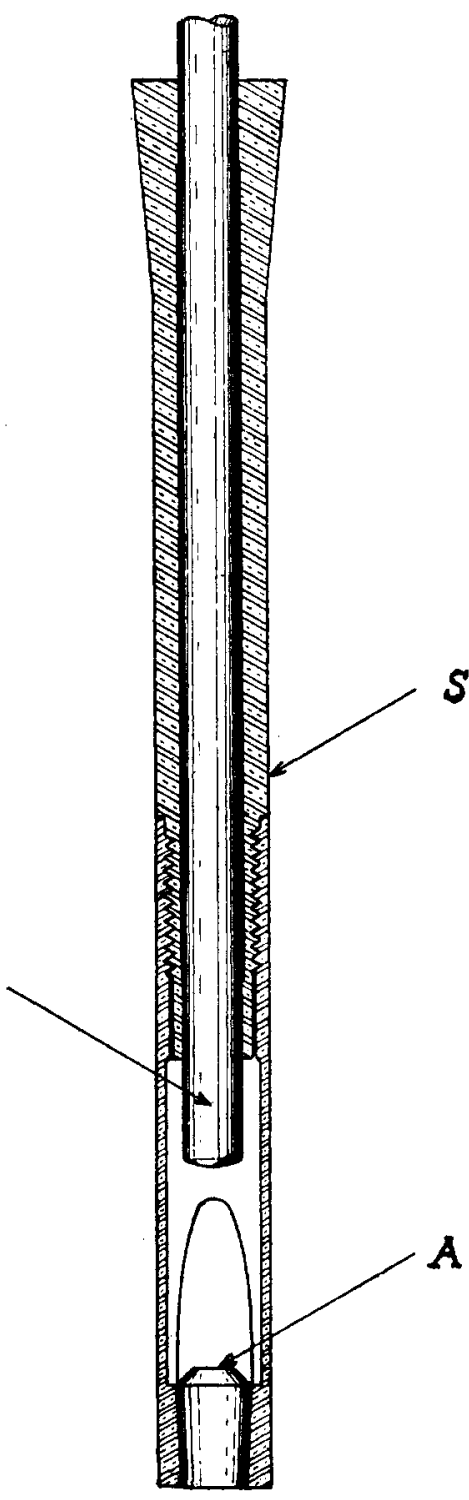

Fig, 2. 
The whole apparatus assembled is shown in Fig. 3. The motor operates a stirrer within the constant temperature bath. The Kipp generator and the train of wash-bottles are for the generation and purification of hydrogen used in stirring the water within the crystallizing tube. This hydrogen was cooled before entering the crystallization tube, by a bath of water and melting ice shown mounted on the same ring stand that sup-

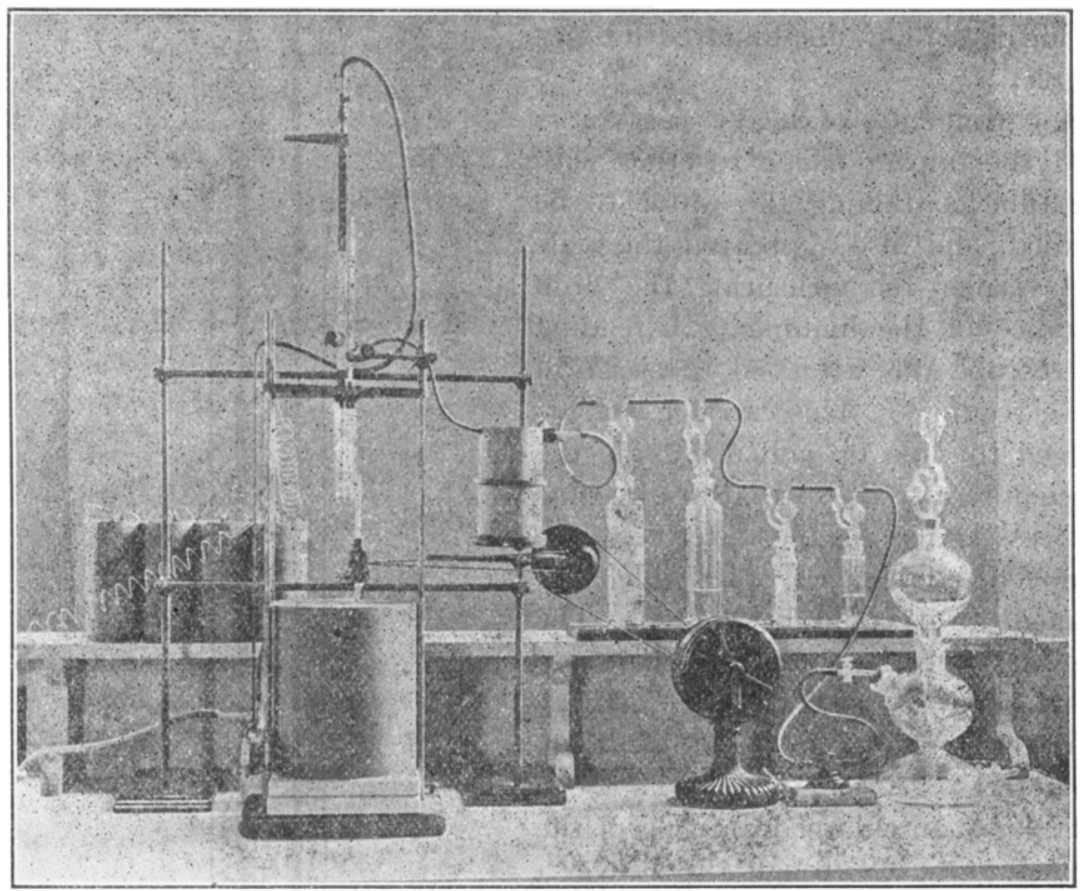

Fig. 3.

ports the transmission gear of the thermostat stirrer. The four dry cells were used to minimize corrosion of the impact parts when these were of steel. Their negative pole was connected with the apparatus, and the positive pole was grounded. A very considerable protection against corrosion was obtained in this way, probably due to leakage current, and no effect on the impact values was found.

The fall distance of the hammer was regulated by a micrometer screw, the latter controlling the releasing device sliding in the ways above the bed. By means of this and the reading microscope, differences of fall as small as $0.05 \mathrm{~mm}$. may be read with certainty.

The thermometer used throughout was one specially made for the purpose, and was standardized against a normal thermometer bearing the certificate of the Reichsanstalt. 
In a part of the experiments the impact parts were made of different kinds of steel. Many difficulties were encountered with these, and they were later replaced by stellite ${ }^{1}$ parts. These showed themselves to be entirely free from corrosion and, having a much less heat conductivity than steel, were preferable for this reason also. One of the chief difficulties in carrying out measurements in the immediate vicinity of the melting point, where very small fluctuations in temperature were of great importance, lay in the conduction of heat to the liquid through the metal parts of the apparatus. With the stellite this was very much minimized.

The water used was redistilled laboratory distilled water. The redistillation was carried out in order to remove any dust particles that it might contain. The water from the redistillation was collected directly in a dust-proof container, from which it was drawn as needed, through a siphon.

The Method of Carrying Out a Determination.--In carrying out a determination, $30 \mathrm{cc}$. of the specially purified water were placed in the crystallization tube, this being sufficient to cover the surface of the anvil about one inch. The carriage was then lowered so that the crystallizing tube was immersed in the thermostat, and the bath was brought to the desired temperature. The hydrogen stirrer was allowed to run for twenty minutes before taking a reading. At the end of this time the flow of hydrogen was stopped and the hammer raised to a small known height, which was thought to be too small to stimulate crystallization upon falling. If upon falling, crystallization did not result, the hammer was raised a small fraction of a millimeter higher than before and allowed to fall again. This operation was repeated until that height sufficient to produce crystallization was found. Similar determinations were then carried out at other temperatures.

\section{Experimental Results.}

The first series of measurements was made with a hammer of carbon steel containing $\mathrm{I} .10 \% \mathrm{C}$ and an anvil of chrome-nickel steel, $\mathrm{Cr}=1.5 \%$, $\mathrm{Ni}=3.5 \%, \mathrm{C}=0.3 \%$. Numerous experiments were also made with manganese steel and with carbon steel that had been differently heat-treated. In all these experiments, however, a rather curious conduct was noticed. It was found that, starting with freshly polished impact parts of any sort whatever, the impact values showed a rapid decrease as time went on. With the freshly polished parts at a given temperature, there would be at first required quite large impact values to produce crystallization. In attempting to attain a duplicate result at the same temperature, it would invariably happen that freezing occurred under a smaller drop

I Generously furnished by Mr. Elwood Haynes, of Kokomo, Indiana, the discoverer of this alloy, to whom our sincere thanks are here expressed. Stellite is an alloy of cobalt and chromium. See J. Ind. \& Eng. Chem., 2, 397. 
than in the first case. This increase in what may be called the sensitiveness of the impact parts would continue for a considerable number of impacts, and then remain fairly constant for a considerable time. Finally, however, the parts would begin to show fluctuating values, and become wholly unreliable.

It was also found in all cases that the increased sensitiveness could bedestroyed by repolishing. Series of consistent results could not be obtained, unless the pair of impact parts was used after its sensitiveness had increased to a maximum and before it began to show the later fluctuating values. This period of constant sensitiveness, while usually not very great, is still great enough to allow of quite a considerable series of measurements being made. The difference between the sensitiveness of a freshly polished pair of impact surfaces and a pair which has acquired sensitiveness by use is very great as may be seen from the following table of results (Table I):

Table I.-Showing Increase in Sensitiveness of Stellite Parts Under Usei. Fali Heights in Militmeters on Successive Determinations.

$\begin{array}{lcccccccccc}\text { No. of det..... } & 1 & 2 & 3 & 4 & 5 & 6 & 7 & 8 & 9 & 10 \\ \text { Fall height.... } & 14.0 & 12.8 & 12.0 & 8.9 & 8.5 & 7.5 & 6.0 & 3.8 & 2.8 & 2.7 \\ \text { No. of det..... } & 11 & 12 & 13 & 14 & 15 & 16 & 17 & 18 & 19 & 20 \\ \text { Fall height.... } & 2.5 & 2.4 & 2.0 & 1.8 & 1.5 & 1.3 & 1.1 & 1.0 & 0.9 & 0.9\end{array}$

All measurements made at $-2.0^{\circ}$.

Two series of measurements, $A$ and $B$, were now undertaken in order to determin the effect of the radius of curvature of the hammer point, that of the anvil being flat. In these two series the parts were repeatedly repolished. A third series was also undertaken (Series C) with parts which had acquired their maximum sensitiveness, and still had not reached the fluctuating stage. In all cases the hammer was of carbon steel and the anvil of chrome-nickel steel. The hammer weight was always 44.5 grams. In Series A the hammer had a conical point, very slightly rounded at the tip. In Series B and C, the radius of curvature of the hammer point was one-fourth of an inch. The results of the three series are represented in the three curves in Fig. 4, the curves being lettered to correspond with the series. Ordinates are drop heights in millimeters, and abscissas are amounts of supercooling in degrees.

The curves approximate somewhat closely to rectangular hyperbolas, although not accurately. As will be seen, the sensitiveness of the sharper point is somewhat less than that of the rounded one, while that of the unrepolished impact parts is very much greater than either.

The Cause of the Increased Sensitiveness of Impact Parts under Use without Repolishing.-The most natural explanation of this phenomenon is that it is due to increased hardness under repeated impacts. In order to test this hypothesis a large number of scleroscopic tests were made on 
the metals used in this work. The instrument used was a Shore Scleroscope ${ }^{1}$ and the method used was to make a large number of determinations of the coefficient of restitution by dropping the hammer of the scleroscope repeatedly on the same spot. Under the repeated dropping of the

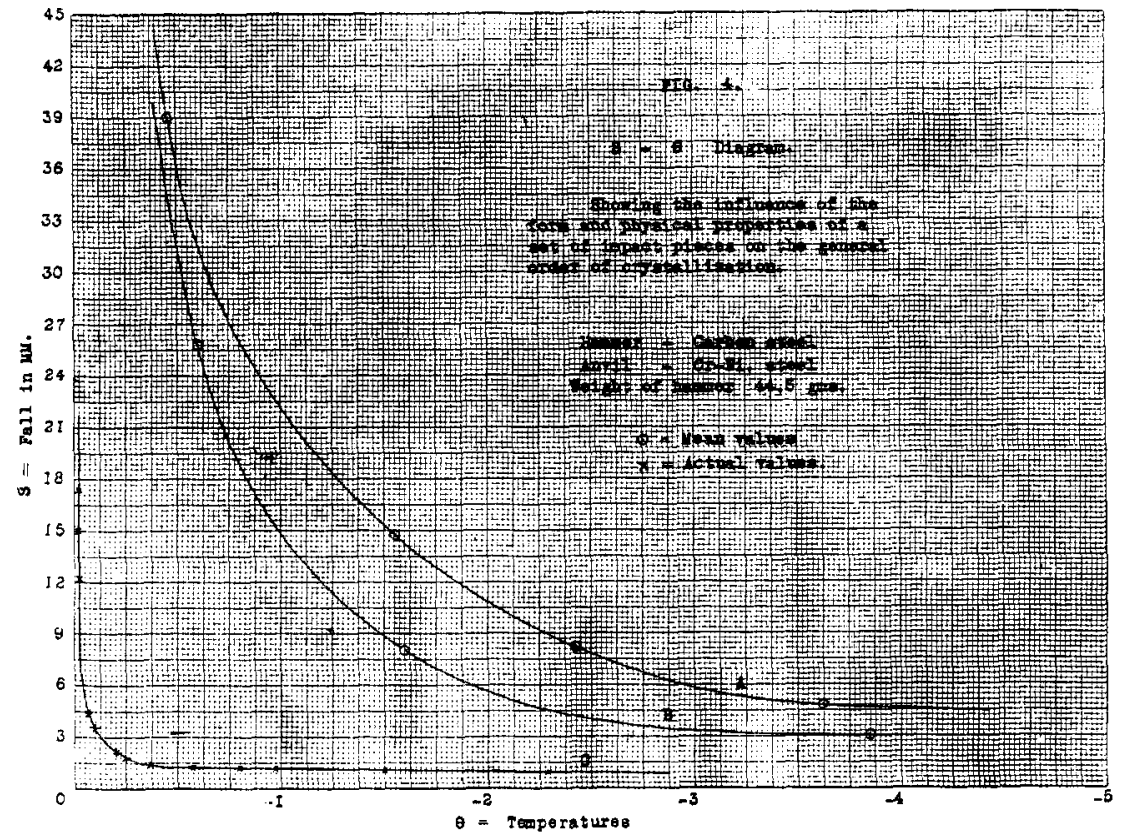

hammer, it should happen that if an increase in hardness occurred, there should also occur an increase in the coefficient of restitution. The curves shown in Figure 5 are the result of these measurements. It will be seen that in every case there is a considerable and rapid increase in hardness for a time, after which it remains roughly constant. That later on fluctuating conditions of hardness raise is shown quite clearly in the case of water-hardened carbon steel, but not so clearly by the others. The short curve $\mathrm{A}$ was obtained by producing each impact in a new spot, and shows the accuracy of the instrument and the uniformity of the metal.

We have also made microscopic examinations of impact surfaces from time to time, and it seems that after a certain amount of use, either minute fractures occur, or else, possibly, some type of crystallization sets in. It seems probable that this fact in connection with the scleroscopic tests offers sufficient explanation of the changes in the value of the sensitiveness of steel and stellite impact parts under use.

1 The sclerosrope was loaned by the Metal Weld Co. of San Francisco, to whom we hereby express our thanks. 
The Degree of Supercooling Necessary before Crystallization can be Forced by Impact. - In the first paper above cited, it was shown that one-tenth of a degree supercooling on the part of water was sufficient to allow of crystallization being forced by impact. With the improved apparatus it was thought that this limit might be considerably reduced. In order to determin this, account must be taken of a certain small error that at-

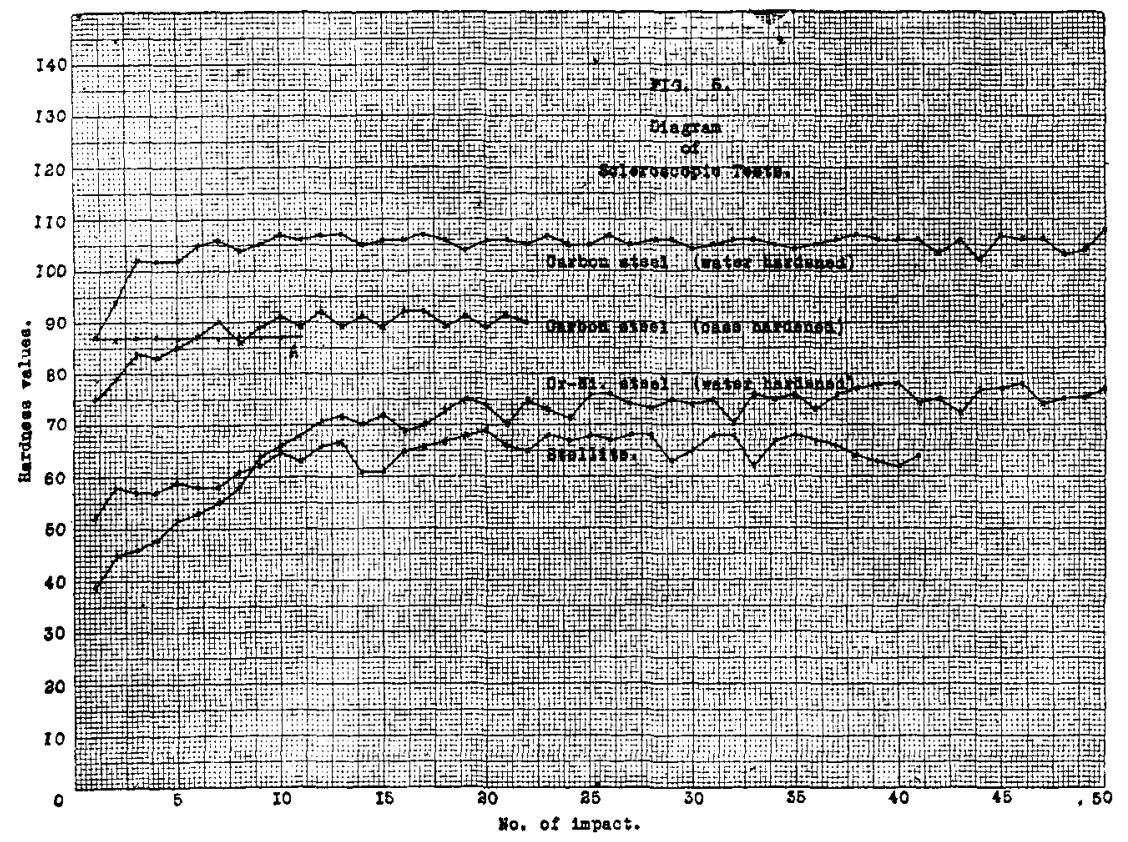

taches to all temperature measurements made throughout the whole investigation. This error is due to the fact that the metal parts of the apparatus which are immersed in the liquid to be frozen are continually conducting heat from the surroundings into the liquid. The result of this is that the liquid at the point of impact is at a somewhat higher temperature than that throughout the body of the liquid. The following method was used to determin the amount of this difference.

The thermometer was placed in its usual position in the apparatus and a steady flow of hydrogen for stirring purposes was established. The temperature in the thermostat was carefully maintained constant throughout the time of the observations. After half an hour or more, this time being allowed for the settling down of the apparatus to constant conditions, the reading of the thermometer was taken. Then, without interrupting the stirring or any of the other conditions, the thermometer was introduced into the metal sleeve, thus occupying the position usually occupied by the hammer rod. After another half hour the reading of the thermometer was 
again taken, and was found to be $0.065^{\circ}$ higher than the first reading. Ten such measurements were made, and all of them agreed with one another within the limits of reading the thermometer, which were $\pm 0.005^{\circ}$. This of course gives the result within the sleeve, and not the actual value at the point of impact, which is undoubtedly somewhat higher still, but there seems to be no simple method of getting at this latter value. Using the correction of $0.065^{\circ}$, we were able to freeze water repeatedly at a temperature slightly under two one-hundredths of one degree below its melting point. In order to freeze water at this temperature, the blow delivered by a 37 -gram stellite hammer upon a stellite anvil with a drop height of eleven millimeters was sufficient, provided that the impact parts had acquired high sensitiveness by repeated use. Thus, if water possesses a metastable limit, it lies at less than two one-hundredths of a degree below its melting point.

The Relation between Energy of Impact and Supercooling.-Two complete series of measurements were now undertaken in order to determin as far as possible the quantitative relationship between the energy o impact and the degree of supercooling. In Fig. 6 is shown a curve obtained

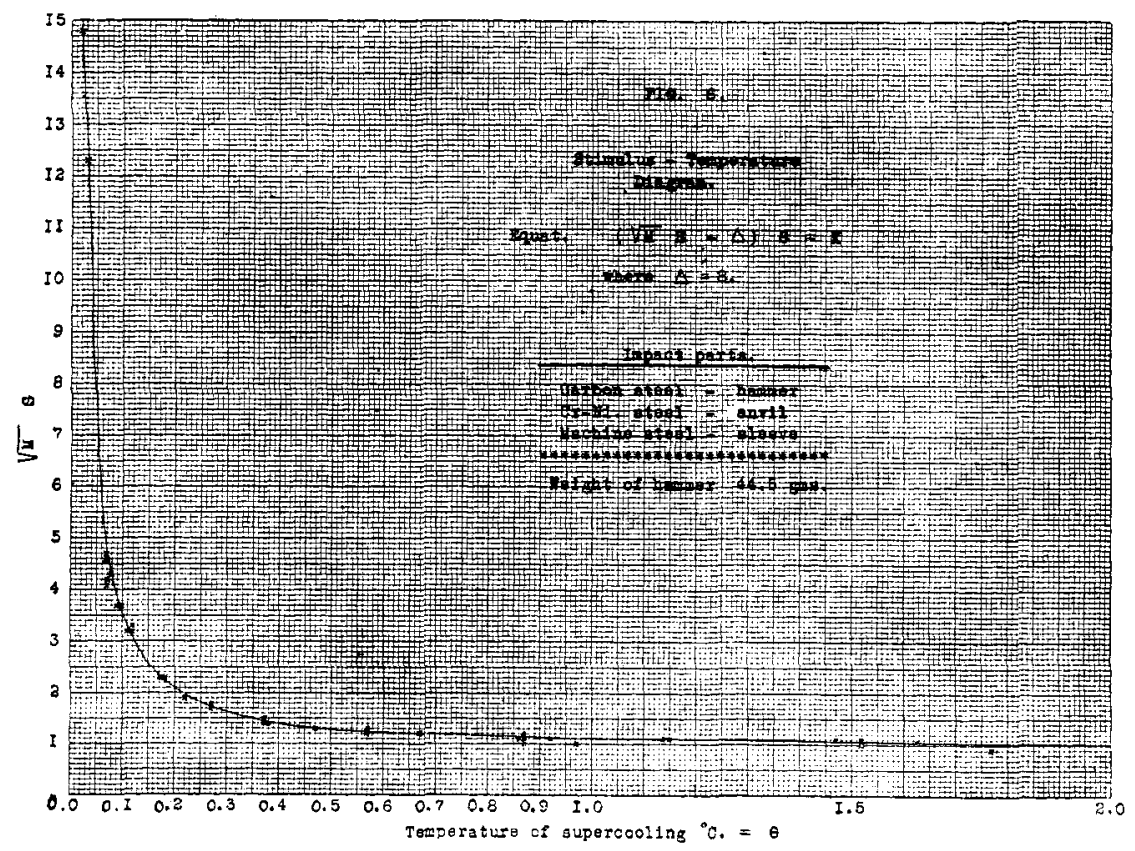

using a carbon steel hammer and a chrome-nickel steel anvil. Abscissas are $\theta$-values, that is, degrees of supercooling. As ordinates are plotted the values of the fall-height in millimeters, multiplied by the square root of the mass of the hammer. The reason for using the square root of the 
mass instead of the mass itself will appear shortly. In Fig. 7 is shown a curve obtained by using stellite impact parts. The impact parts were brought to a condition of maximum sensitiveness before beginning the measurements, and the whole set of measurements was finished before the fluctuating state had set in.

A comparison of the two curves will show that the stellite parts are less efficient in forcing crystallization than are the steel parts, that is, at a given temperature, a heavier blow is required with stellite than with

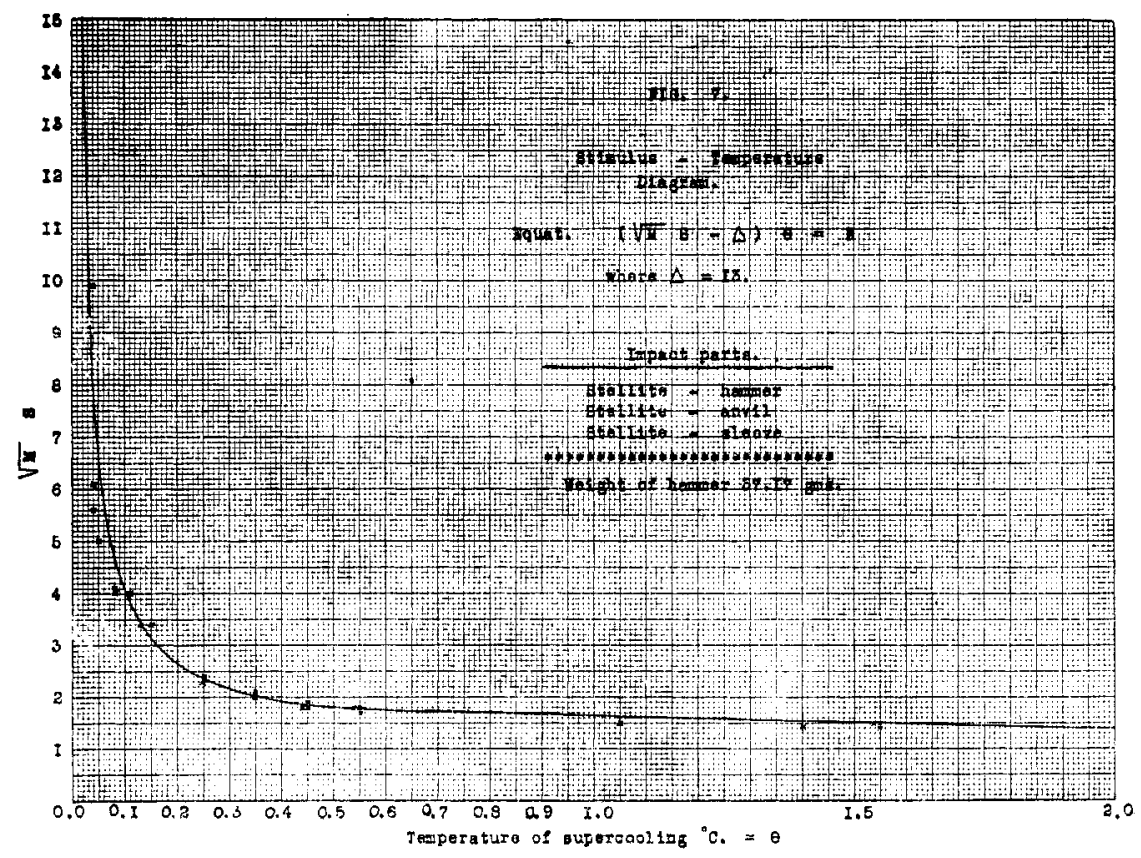

steel. This is undoubtedly due to the fact that the steel is considerably harder than the stellite (see scleroscopic tests). In fact, the relative effects are fairly closely proportional to the relative coefficients of restitution of the metals used. At any rate the coefficient of restitution manifestly plays an important part in determining the activity of the impact parts. If the above surmise that the activity is proportional to this property is correct, it would follow that with bodies whose coefficient of restitution was unity, that is, with perfectly elastic bodies as impact parts, the crystallization stimulus would be otherwise independent of the nature of the bodies. In the curves given, the approximation to rectangular hyperbolas is quite close, but the curves are considerably elevated above the $\mathrm{X}$-axis. The extent of this elevation is greatest in the case of stellite, and the values of the displacement in the two cases are roughly inversely proportional to the coefficients of restitution in the two cases. In the 
drawing of the curves, however, this upward displacement has been treated as an additive value, and is indicated by $\Delta$. The line curve is plotted from the following equation:

$$
(\sqrt{M} \times S-\Delta) \Theta=\text { constant, }
$$

where $M$ is the mass of the hammer in grams, $S$ is the fall distance in centimeters, and $\Theta$ is the supercooling in degrees. For the steel parts $\Delta$ is taken as 8 , and for stellite parts it is taken as 13 . The crosses are the values for actual determinations, and it is readily seen that the agreement with the theoretical is very good.

The Mass Function of the Hammer.-It wonld seem most natural to suppose that the crystallization stimulus resulting from an impact would be determined by the energy developed at a given point as a result of the impact. In this case, what may be called the "stimulus-function" would be proportional to the mass and to the fall distance. While with a given hammer the stimulus is apparently proportional to the fall distance, it is not true that it is proportional to the mass of the hammers when different hammers are used. The following results show this quite clearly. The temperature was $-0.25^{\circ}$. The numbers are fall distances in centimeters required to produce crystallization in the case of the two hammers whose weights are specified:

Weight of hammers in grams.

\begin{tabular}{|c|c|c|}
\hline & \\
\hline & 72.45 & 37.17 \\
\hline Fall in centimeters $\ldots \ldots \ldots \ldots \ldots$ & 0.225 & 0.305 \\
\hline Fall in centimeters $\ldots \ldots \ldots \ldots \ldots$ & 0.205 & 0.305 \\
\hline Fall in centimeters $\ldots \ldots \ldots \ldots \ldots$ & 0.225 & 0.305 \\
\hline Average. . & 0.218 & 0.305 \\
\hline
\end{tabular}

If the stimulus were proportional to the mass we should have at a fixed temperature

$$
\mathrm{M}_{1} \mathrm{~S}_{1}=\mathrm{M}_{2} \mathrm{~S}_{2} \text {, }
$$

since the stimulus has always been found proportional to S. Substituting values given in the above table we get:

$$
\mathrm{M}_{1} \mathrm{~S}_{1}={ }_{15} .9 ; \quad \mathrm{M}_{2} \mathrm{~S}_{2}={ }_{\mathrm{II} .3} .
$$

If, however, we use the square root of the mass instead of the mass, we get much better agreement:

$$
\sqrt{\mathrm{M}_{1}} \times \mathrm{S}_{1}=\mathrm{I} .86 ; \quad \overrightarrow{\mathrm{M}}_{2} \times \mathrm{S}_{2}=\mathrm{I} .86 .
$$

This result seems to be rather irrational, and we prefer to let it stand for the present as an apparent empirical fact. It is hoped that, in the near future, the work will be continued with apparatus which will yield more certain results. The frequent and sudden development of fluctuations in the activity of the metal impact parts makes long continued work with definit conditions impossible. We are arranging for the construction of stellite impact parts in which will be set carbonados, or Brazilian dia- 
monds at the point of impact, with which it is hoped that many of the difficulties with which we have had to contend will disappear.

Stanford University, Caz., June 5, 1913.

[CONTRIBUTION FROM THE JOHN HARRISON LABORATORY OF ChEMISTRY.]

\section{HALIDE BASES OF COLUMBIUM.}

BY HERBERT S. HARNED.

Received July 23, 1913.

In 1907 Chabriè 1 reported a lower hydrated chloride of tantalum to which he gave the formula $\mathrm{TaCl}_{2} \cdot 2 \mathrm{H}_{2} \mathrm{O}$. He obtained this substance by heating tantalum pentachloride with sodium amalgam in a tube of hard glass, which was sealed at one end and evacuated by means of a suction pump.

In 1908, Van Haagen, ${ }^{2}$ working with the halides of tantalum and their products of reduction, got, by passing tantalum pentabromide through a red hot tube in a current of hydrogen, a greenish black substance which was soluble in water to an intense emerald-green solution. Every attempt to produce by this method a sufficient quantity of material for investigation proved of no avail.

During the winter of 1908-1909, Chapin ${ }^{3}$ continued this work. He used the Chabriè method of reduction on tantalum pentabromide. The reaction mass was boiled with water containing a little hydrobromic acid. An intense emerald-green solution similar to that described by Van Haagen was obtained and, on evaporation, black crystals separated. These were filtered off and dried. When ground to a powder, this substance had a green color. It was very soluble in water, the solution possessing a remarkable coloring power ( $\mathrm{I}$ gram in $100 \mathrm{cc}$. of water giving such an intense coloration that a layer half an inch thick was opaque). Ammonia water precipitated a brown hydroxide from this solution.

Chapin carried out a number of analyses which showed that this compound possessed a composition represented by the formula $\left(\mathrm{Ta}_{6} \mathrm{Br}_{12}\right)$ $\mathrm{Br}_{2} \mathrm{H}_{2} \mathrm{O}$. Molecular-weight determinations by both the freezing- and boiling-point methods also indicated this constitution. Only one-seventh of the bromine acted as ion. The tantalum seemed to be present in a complex cation.

From this compound Chapin prepared an interesting series of derivatives.

He also made Chabriè's chloride from tantalum pentachloride and proved that it was $\left(\mathrm{Ta}_{6} \mathrm{Cl}_{12}\right) \mathrm{Cl}_{2 .}{ }_{7} \mathrm{H}_{2} \mathrm{O}$.

The object of the present investigation was to find out whether these

1 Compt. rend., 144, 804 (1907).

2 Thesis, University of Pennsylvania, 1908.

- This Journal, 32, 323 (Igio). 